\title{
Study on dynamics of structural transformation during charge/discharge of $\mathrm{LiFePO}_{4}$ cathode
}

\author{
Hao-Hsun Chang ${ }^{\mathrm{a}}$, Chun-Chih Chang ${ }^{\mathrm{a}}$, Hung-Chun Wu ${ }^{\mathrm{b}}$, Mo-Hua Yang ${ }^{\mathrm{b}}$, \\ Hwo-Shuenn Sheu ${ }^{\mathrm{c}}$, Nae-Lih $\mathrm{Wu}^{\mathrm{a}, *}$ \\ ${ }^{a}$ Department of Chemical Engineering, National Taiwan University, Taipei 106, Taiwan, ROC \\ ${ }^{\mathrm{b}}$ Materials Research Laboratories, Industrial Technology Research Institute, Chutung, Hsinchu 310, Taiwan, ROC \\ ${ }^{\mathrm{c}}$ National Synchrotron Radiation Research Center, Hsinchu 30077, Taiwan, ROC
}

Received 24 October 2007; received in revised form 14 November 2007; accepted 15 December 2007

Available online 25 December 2007

\begin{abstract}
Structural transformation taking place during charge/discharge of the $\mathrm{LiFePO}_{4}$ electrode in an organic Li-ion electrolyte has been studied by in situ synchrotron X-ray diffraction (XRD) concurrently with electrochemical analysis. The data reveal complex structural transformation patterns which result from significantly delayed structural transformation, even at low to moderate current rates $(\mathrm{C} /$ $10 \sim 1 \mathrm{C}$ ). The extent of the deviation is affected by charge/discharge conditions as well as history, and strong deviation appears detrimental to electrode cycle life. Furthermore, the two-phase characteristic of the $\mathrm{Li}^{+}$insertion/extraction reactions is found to persist even within one metastable crystal structure. The result suggests the presence of domain structures that are probably caused by the strong electron/ion site coulombic interaction as previously suggested.
\end{abstract}

(C) 2007 Elsevier B.V. All rights reserved.

Keywords: $\mathrm{LiFePO}_{4}$; Structural transformation; Synchrotron X-ray diffraction; Li-ion secondary battery; Cathode

\section{Introduction}

$\mathrm{LiFePO}_{4}$ has drawn intensive attention in recent years for its potential application as a cathode material for high-power rechargeable Li-ion batteries [1-6]. Several reports have demonstrated high-rate capability $(>10 \mathrm{C})$ with high coulombic efficiency $(>80 \%)$ and satisfactory cycle life [7-9]. To date, the crystallographic properties and phase-stoichiometry relation of the $\mathrm{Li}_{1-\Delta x} \mathrm{FePO}_{4}$ system have been thoroughly studied $[10,11]$. The crystal structure of $\mathrm{LiFePO}_{4}$ can be described by space group Pmna and is composed of corner-sharing $\mathrm{FeO}_{6}$ octahedra forming two-dimensional square lattice perpendicular to the $a$-axis, edge-sharing $\mathrm{LiO}_{6}$ aligned along the $b$-axis, and $\mathrm{PO}_{4}$ groups connecting neighboring planes. The structure of the delithiated phase, $\mathrm{FePO}_{4}$, is described by the

\footnotetext{
* Corresponding author. Tel.: +8862 23627158; fax: +886223623040. E-mail address: nlw001@ntu.edu.tw (N.-L.Wu).
}

same space group but with distinctly different lattice parameters. Yamada et al. [12] studied on the room-temperature phase behavior of $\mathrm{Li}_{1-\Delta x} \mathrm{FePO}_{4}$ system with $0 \leqslant \Delta x \leqslant 1$ by progressive chemical extraction of Li-ions from $\mathrm{LiFePO}_{4}$, and their data showed that there does not exist stable intermediate compound except for two small regions $(<5.0 \mathrm{~mol} . \%$ in $\mathrm{Li}$ content $)$ of solid solutions toward the end compounds. (The small solid-solution ranges will hereafter be neglected for simplicity in discussion.) Study by Delacourt et al. [13] on the phase behavior by thermal annealing basically confirmed the same phase relation at temperatures below $50^{\circ} \mathrm{C}$. Solid-solution over entire Li stoichiometry range $(0 \leqslant \Delta x \leqslant 1)$ was, on the other hand, found to exist above $350{ }^{\circ} \mathrm{C}$.

It is noted that the phase-structure-stoichiometry relations have so far been established mainly by non-electrochemical methods. It is intriguing to know how well the structural transformation during electrochemical lithiation/ delithiation of $\mathrm{LiFePO}_{4}$ actually follows the established 
relations and how the deviation, if any, could affect the performance of the $\mathrm{LiFePO}_{4}$ electrode in practical applications.

To answer these questions, structural transformation during electrochemical lithiation/delithiation of the LiFe$\mathrm{PO}_{4}$ electrode was studied in this work by using in situ synchrotron X-ray diffraction (XRD). The results revealed complex structural transformation dynamics and unique characteristics of the two-phase reaction nature of this electrochemical system.

\section{Experimental}

Commercial $\mathrm{LiFePO}_{4}$ powder (Phostech) is used as received. The powder has a particle size of $d_{50}=3.0 \mu \mathrm{m}$. $\mathrm{LiFePO}_{4}$ electrodes were made of $85 \mathrm{wt} . \% \mathrm{LiFePO}_{4}$, 5 wt.\% graphitic flakes (KS6, $3 \mu \mathrm{m}$, TIMCAL), $2 \%$ nanosized carbon black (Super P, $40 \mathrm{~nm}$, TIMCAL) and 8 wt. \% organic binder (polyvinylidene difluoride; Aldrich) with $\mathrm{Al}$ foil as the current collector. The resulting $\mathrm{LiFePO}_{4}$ electrode was assembled together with a Li-foil counter electrode to make either coffee-bag cells or CR2032 coin cells, which use electrolyte of $1 \mathrm{M} \mathrm{LiPF}_{6}$ in a 1:2 v/v mixture of ethylene carbonate (EC) and ethyl methyl carbonate (EMC; Mitsubishi Chemical). All the cells were assembled in a dry room where the dew point was maintained at between -40 and $-45^{\circ} \mathrm{C}$.

In situ synchrotron XRD was conducted by using beamline 01-C2 in National Synchrotron Radiation Research Center, Taiwan, ROC, and an X-ray source of $0.10223 \mathrm{~nm}$ in wavelength was employed. XRD spectra were acquired during the charge/discharge tests carried out at $55^{\circ} \mathrm{C}$.

\section{Results and discussion}

Fig. 1a shows the synchrotron XRD patterns of selected $2 \theta$ range acquired during a discharge/charge cycle under $1 \mathrm{C}$ rate with an intermittent rest period, while the corresponding voltage curve is shown in Fig. 1b. The structure transformation can be monitored by, for instance, following the evolution of the (211) and (020) reflection peaks of the end compounds, as marked in Fig. 1a. As shown, the XRD pattern show only the $\mathrm{FePO}_{4}$ structure before the 8th measurement during discharge, and then transformed rapidly into that of the $\mathrm{LiFePO}_{4}$-structure during the period between the 9th and 11th measurements, when the test was switched to rest. The inset shown in Fig. 1a plots the intensities of the $(020)$ peaks of the $\mathrm{LiFePO}_{4}$ and $\mathrm{FePO}_{4}$-structures, , confirming the delayed $\mathrm{FePO}_{4}$-to$\mathrm{LiFePO}_{4}$ transformation until near the end of discharge.

Similarly, during charge, the crystal structure of the electrode remained as the $\mathrm{LiFePO}_{4}$-structure until near the end of charge, and then transformed abruptly to the $\mathrm{FePO}_{4}$ structure upon reaching another rest period (Fig. 1a and b). It is important to note that, both the discharge and charge voltage curves (Fig. 1b) exhibit a flat-plateau region, where the XRD patterns show essentially only one struc- a
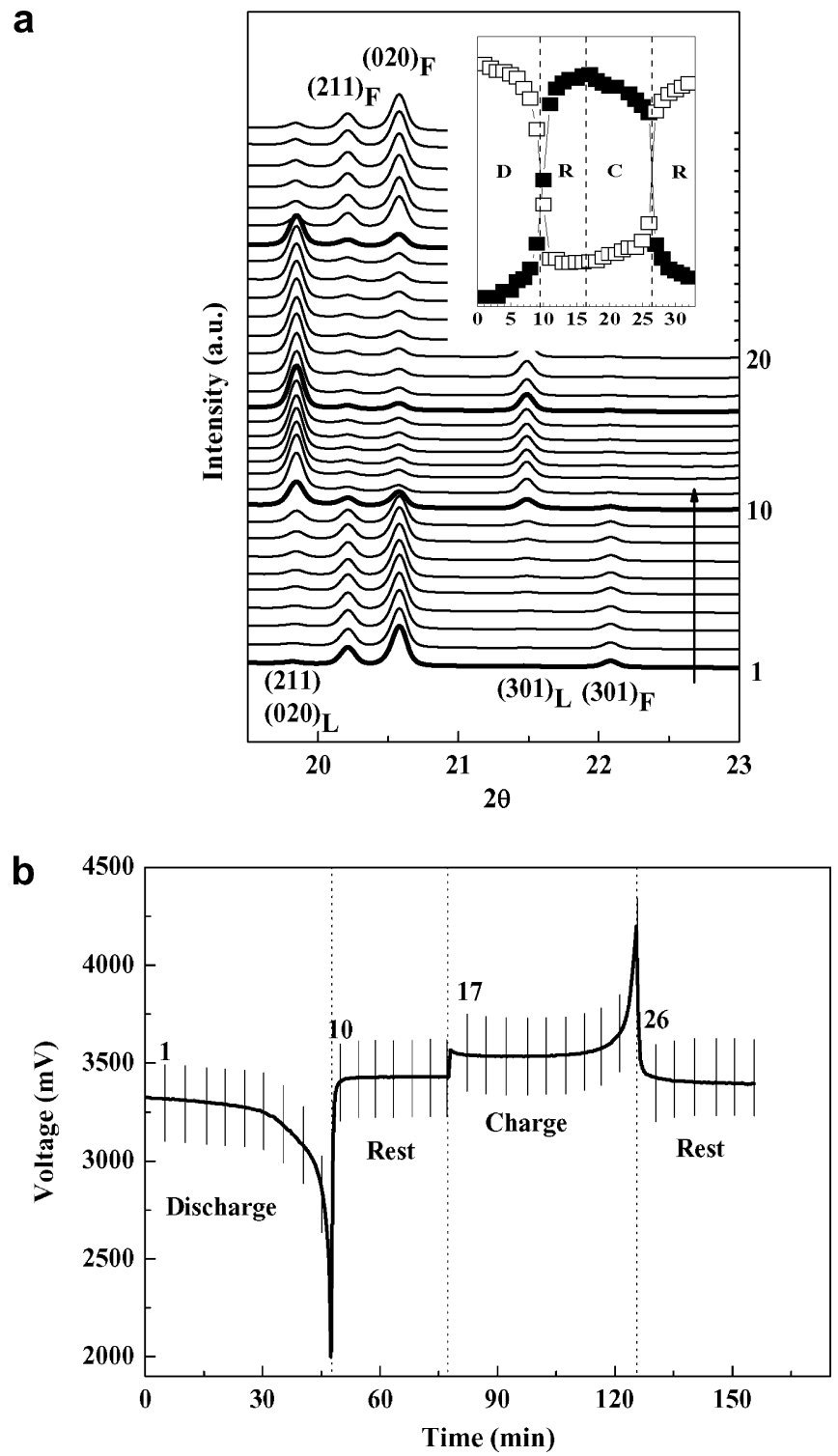

Fig. 1. XRD and electrochemical data for charge/discharge test of Cell \#1 at $1 \mathrm{C}$ rate at $55^{\circ} \mathrm{C}$ with an intermittent rest period. (a) Synchrotron XRD patterns. The subscripts $\mathrm{F}$ and $\mathrm{L}$ indicate, respectively, reflections from the $\mathrm{FePO}_{4}$ - and $\mathrm{LiFePO}_{4}$-structures. The bold curves mark the onset of different stages ( $\mathrm{C}$ for charge; $\mathrm{D}$, discharge; $\mathrm{R}$, rest) within a test cycle. The inset shows the XRD peak intensities of $\mathrm{LiFePO}_{4}(020)(\boldsymbol{\square})$ and $\mathrm{FePO}_{4}$ (020) ( $\square$ ) versus the measurement number. (b) Voltage curve. The numbers shown along the right-axis in (a) and within the figure in (b) index the XRD measurement number.

ture. A plateau typically observed over the middle capacity region of an either charge or discharge voltage curve has been considered as a signature of the two-phase reaction nature, namely

$\mathrm{LiFePO}_{4} \leftrightarrow \mathrm{e}^{-}+\mathrm{Li}^{+}+\mathrm{FePO}_{4}$

of the present electrochemical system $[1,12,14]$.

Delayed structural transformation was also detected even at $\mathrm{C} / 10$ rate at room temperature (not shown). Under such a condition, transformation from the $\mathrm{LiFePO}_{4}$-structure to the $\mathrm{FePO}_{4}$-structure did not commence until $\sim 70 \%$ 
of Li-ion was extracted. Again, the characteristic of the voltage flat-plateau was also observed during the "structure-frozen" period. It is worth noting that the "frozen" structures are different from structures of solid-solutions. For the latter, the lattice parameters change progressively with the stoichiometry, while the lattice parameters simply remain unchanged for the former.

Fig. 2 shows the result of the first cycle of another cell (Cell \#2) which was tested without intermittent rest. There
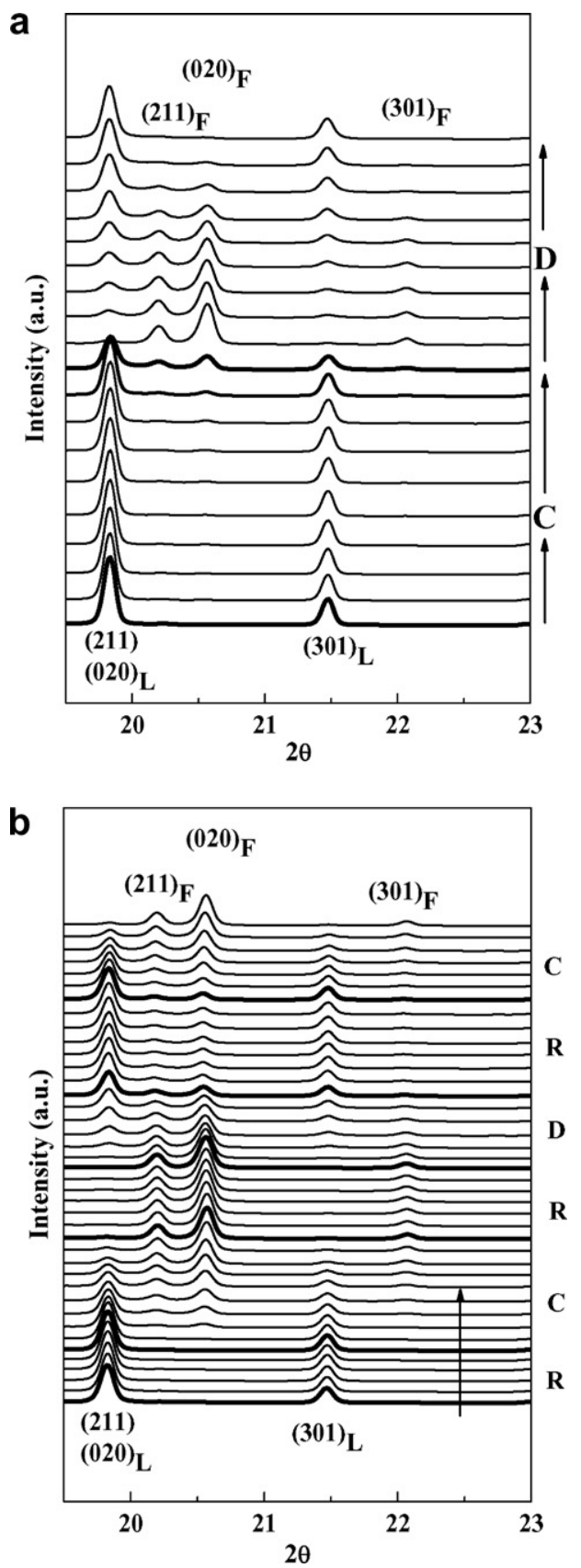

Fig. 2. XRD data for Cell $\# 2$ at $1 \mathrm{C}$ rate at $55^{\circ} \mathrm{C}$ : (a) the first cycle without intermittent rest; (b) the second and third cycles with intermittent rest. The subscripts $\mathrm{F}$ and $\mathrm{L}$ indicate, respectively, reflections from the $\mathrm{FePO}_{4-}$ and $\mathrm{LiFePO}_{4}$-structures. The bold curves mark the onset of different stages ( $\mathrm{C}$ for charge; $\mathrm{D}$, discharge; $\mathrm{R}$, rest) within a test cycle. was essentially no structure change during charge (Fig. 2a), similar to the charge period shown in Fig. 1a. However, as there was no intermittent rest in this case, structure transformation from the $\mathrm{LiFePO}_{4}$-structure to the $\mathrm{FePO}_{4}$-structure actually took place during discharge (lithiation)! The XRD peak intensities of the $\mathrm{FePO}_{4}$-structure reach the maximum sometime during the discharge period. In spite of such a complex structural transformation process, the voltage curves showed the same flat-plateau characteristic as seen in Fig. $1 b$.

It was, nevertheless, found that, after the non-interrupting first cycle, the structural transformation started to proceed "normally" in the subsequent cycles either with or without intermittent resting between charge and discharge (Fig. 2b). For a "normal" structure transformation process, the extent of the transformation, as indicated by the XRD peak intensities, is correlated almost linearly with the extent of Li-ion extraction or insertion.

The results described above point out two important conclusions. Firstly, structural transformation during charge/discharge of $\mathrm{LiFePO}_{4}$ more than often proceeds in ways that deviate far from the equilibrium phase-structure-stoichiometry relations. The extent of the deviation depends not only on the test condition but also on the charge/discharge history that the electrode has previously experienced. Secondly, the voltage curves consistently show the characteristics of the two-phase mechanism, irrespective of the structural transformation pattern and crystal structure.

In the cases of the delayed structural transformation, it is noted that transformation has been greatly accelerated when there is strong disturbance during the charge/discharge process, such as the sudden stop for the intermittent rest period in Fig. 1 and the lithiation/ delithiation reversal in Fig. 2a. This may explain the fact that the frozen-structure phenomenon has never been detected in the previous ex situ XRD or TEM studies. From the viewpoint of nucleation theory, these observations may suggest that the delay in the transformation result from slow nucleation kinetics of the resulting phase, and the process disturbances tend to stimulate the formation of such nuclei. This assumption may also explain why the transformation process is history-dependent, as different test protocols could result in particular microstructures that serve as favorable nucleation sites. An example is when the $\mathrm{LiFePO}_{4}$ electrode experienced the overlapped structural transformation processes during discharge shown in Fig. 2a. The overlapped transformation process might have introduced microstructures that facilitate nucleation in the subsequent cycles, which show exclusively "normal" transformation process (Fig. 2b). The results also suggest that a few initial non-stopping cycles at moderate to high rates may be a good exercise in practical applications to avoid delayed structure transformation, which, as shown below, could otherwise have adverse effects on the cycling performance of the electrode. 
The effect of the non-equilibrium structural transformation on the performance of $\mathrm{LiFePO}_{4}$ electrode was preliminarily assessed by conducting prolonged cycling tests on two coin cells which have the same electrode compositions as in the coffee-bag cells for the synchrotron studies. Cell \#3 was cycled at $1 \mathrm{C}$ rate at $55^{\circ} \mathrm{C}$ always with an intermittent rest period between charge and discharge, while Cell \#4 was cycled under the same condition but without any intermittent rest period. According to the XRD data shown above, it is anticipated that structural transformation occurs abruptly only near the end of every charge or discharge period for Cell \#3. On the other hand, transformation following closely the equilibrium phase relation is expected from the second cycle up for Cell \#4. Fig. 3 summarizes the discharge capacities versus cycle number for these two cells. Cell \#3 was found to exhibit much worse performance, including lower discharge capacities during initial cycles and faster capacity fading, than Cell \#4. The results suggest that structural transformation that strongly deviates from the equilibrium phase-stoichiometry relation has profound adverse effects on electrode performance. Systematic research is currently underway for understanding the interplay among the structural transformation process, initial discharge capacity and the fading mechanism.

The presence of the voltage plateau suggest that some sort of two-"phase" relation always be maintained within the crystallite, irrespective of the crystal structure. It may be possible to understand this phenomenon based on the theoretical study by Zhou et al. [15] on the phase stability of $\mathrm{LiFePO}_{4} / \mathrm{FePO}_{4}$. By using first-principle calculation, it was demonstrated in their study that electron localization and charge ordering at $\mathrm{Fe}$-ion sites play a very important role in the phase relation of $\mathrm{LiFePO}_{4}-\mathrm{FePO}_{4}$; strong electron/site coulombic interaction becomes a key factor that forbids the formation of continuous solid solution in the binary system. To explain the present observations, it is

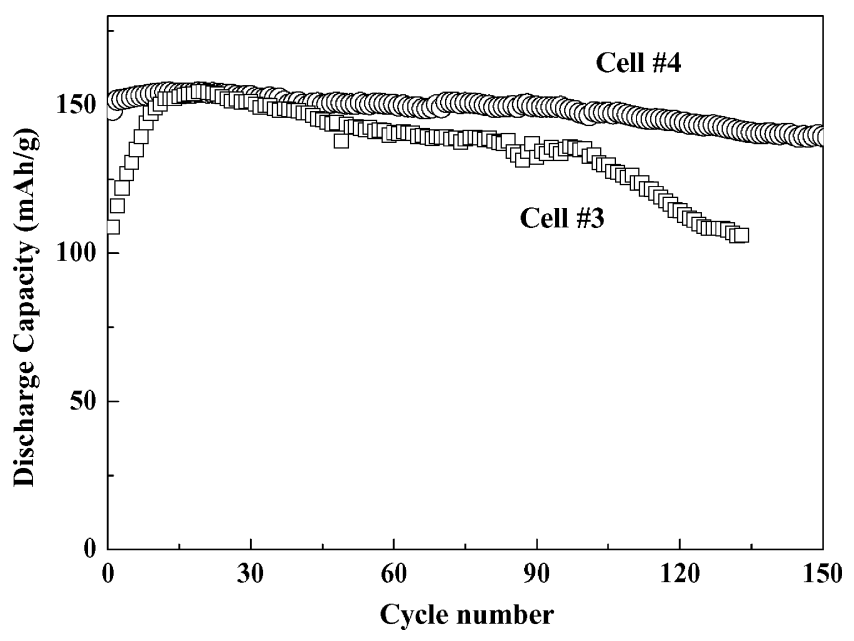

Fig. 3. Discharge capacity versus cycle number. Cell \#3: coin-cell cycled with intermittent rest between charge and discharge; Cell \#4: coin-cell cycled without intermittent rest $\left(1 \mathrm{C}\right.$ rate, $\left.55^{\circ} \mathrm{C}\right)$. proposed that, owing to the strong localized charge interaction, Li-ion extraction do not occur randomly even within the frozen singled-structured crystallites, but rather two"phase" interface be maintained at the boundaries between "Li-rich" and "Li-lean domains which are, respectively, stabilized and energetically differentiated mainly by the coulombic interaction energy difference.

Recently studies by Chen et al. [16] and Laffont et al. [17] using high-resolution electron microscopy and energy loss spectroscopy provide insight into the $\mathrm{LiFePO}_{4}$ $\mathrm{FePO}_{4}$ phase transition at the microscopic level. The lithiation/delithiation was carried out either with chemical means or with electrochemical charge/discharge at very low rate $(\mathrm{C} / 50)$. The data in both studies suggested that, during lithiation/delithiation, Li-ions diffuse in a direction parallel to the $b$-axis of the olivine crystallites, while the phase boundary progresses parallel to the $a$-axis. The data of Laffont et al. [17] further indicate the presence of sharp boundaries between $\mathrm{LiFePO}_{4}$ and $\mathrm{FePO}_{4}$-structure domains within the crystallites. Our presumption of the two-phase equilibrium maintained at the boundaries between the compositional domains is to some extent in line with the interface model of Laffont et al., only that the interface within one lattice structure in the presence study is merely metastable.

In summary, in situ synchrotron XRD study has revealed the complex nature of the transformation dynamics during charge/discharge of $\mathrm{LiFePO}_{4}$ electrode, which cannot be predicted from current understanding of the equilibrium phase relations. The transformation dynamics, in turn, could have a significant impact on the cycling performance of the electrode. Comparison between the XRD and electrochemical data have indicated that the two-phase reaction nature of the electrochemical lithiation/delithiation processes is not governed by crystallographic consideration but more likely by localized electron/ion site coulombic interaction.

\section{Acknowledgments}

This work is partially supported by Industrial Technology Research Institute and by National Taiwan University (contract number 95R0066-BE04-01).

\section{References}

[1] A.K. Padhi, K.S. Nanjundaswamy, J.B. Goodenough, J. Electrochem. Soc. 144 (1997) 1188.

[2] J. Chen, M.S. Whittingham, Electrochem. Commun. 8 (2006) 855.

[3] H. Huang, S.-C. Yin, L.F. Nazar, Electrochem. Solid-State Lett. 4 (2001) A170.

[4] S.-Y. Chung, J.T. Bloking, Y.-M. Chiang, Nature Mater. 1 (2002) 123.

[5] H.C. Wu, C.Y. Su, D.T. Shieh, M.H. Yang, N.L. Wu, Electrochem. Solid-State Lett. 9 (2006) A537.

[6] H.H. Chang, C.C. Chang, H.C. Wu, Z.Z. Guo, M.H. Yang, Y.P. Chiang, H.S. Sheu, N.L. Wu, J. Power Sources 158 (2006) 550.

[7] A.S. Gozdz, A.C. Chu, Y.M. Chiang, G.N. Riley, Jr., US patent application \#2005/0233219A1. 
[8] H.C. Wu, C.Y. Su, P.H. Hsieh, B.M. Lin, M.Y. Yang, N.L. Wu, Taiwan patent \#I270994; US patent application \#US2007/ 0154809A1.

[9] B. Kang, G. Cedar, International Meeting on Lithium Batteries, Biarritz, France, 18-23 June, 2006, paper \#162.

[10] A.S. Andersson, J.O. Thomas, J. Power Sources 97 (2001) 498.

[11] C. Delacourt, J. Rodriguez-Carvajal, B. Schmitt, J.M. Tarascon, C. Masquelier, Solid State Sci. 7 (2005) 1506.

[12] A. Yamada, H. Koizumi, N. Sonoyama, R. Kanno, Electrochem. Solid-State Lett. 8 (2005) A409.
[13] C. Delacourt, P. Poizot, J.M. Tarascon, C. Masquelier, Nature Mater. 4 (2005) 254.

[14] N. Meethong, H.Y.S. Huang, W.C. Carter, Y.M. Chiang, Electrochem. Solid-State Lett. 10 (2007) A134.

[15] F. Zhou, C.A. Marianetti, M. Cococcioni, D. Morgan, G. Ceder, Phys. Rev. B 69 (2004) 201101.

[16] G. Chen, X. Song, T.J. Richardson, Electrochem. Solid-State Lett. 9 (2006) A295.

[17] L. Laffont, C. Delacourt, P. Gibot, M.Y. Wu, P. Kooyman, C. Masquelier, J.M. Tarascon, Chem. Mater. 18 (2006) 5520. 